pÿHeavy metal toxicity and mortality association between density of heavy metal bands and cause specific hospital admissions and mortality : population based cohort study

\title{
Martikainen, Pekka
}

2021-12-15

Martikainen , P , Korhonen , K \& Tarkiainen , L 2021, ' Heavy metal toxicity and pÿmortality association between density of heavy metal bands and cause specific hospital admissions and mortality : population based cohort study ', BMJ : British Medical Journal , vol. 375 , no. (8319) , e067633 . https://doi.org/10.1136/bmj-2021-067633

http://hdl.handle.net/10138/338980

https://doi.org/10.1136/bmj-2021-067633

cc_by_nc

publishedVersion

Downloaded from Helda, University of Helsinki institutional repository.

This is an electronic reprint of the original article.

This reprint may differ from the original in pagination and typographic detail.

Please cite the original version. 

and mortality: population based cohort study

\author{
Pekka Martikainen, ${ }^{1,2,3}$ Kaarina Korhonen, ${ }^{1}$ Lasse Tarkiainen ${ }^{1}$
}

\begin{abstract}
${ }^{1}$ Population Research Unit,
Faculty of Social Sciences,

University of Helsinki, Helsinki,

Finland

${ }^{2}$ Max Planck Institute for

Demographic Research, Rostock Germany

${ }^{3}$ Department of Public Health Sciences, Stockholm University,

Stockholm, Sweden

Correspondence to:

P Martikainen

pekka.martikainen@helsinki.fi

(ORCID 0000-0001-9374-1438)

Additional material is published

online only. To view please visit

the journal online.

Cite this as: BMJ2021;375:e067633 http://dx.doi.org/10.1136/

bmj-2021-067633
\end{abstract}

Accepted: 27 October 2021

\section{ABSTRACT}

OBJECTIVE

To assess the association between area level density of heavy metal bands and cause specific hospital admissions and mortality.

\section{DESIGN}

Longitudinal register based cohort study.

SETTING

311 municipalities in Finland.

PARTICIPANTS

3644944 people aged 15 to 70 residing in Finland at the end of 2001 .

\section{MAIN OUTCOME MEASURES}

Hospital admission and mortality from all causes, internal causes, alcohol attributable causes, accidental injury and violence, suicide or self-harm, and mental health related causes. Appendicitis and toxic effects of metals were negative control outcomes.

\section{RESULTS}

During 50.4 million person years of follow-up in 2002-17, 4237807 person years with hospital admissions were observed and 221912 individuals died. Mortality in municipalities with a moderate density of heavy metal bands ( $<5.7$ per 10000 inhabitants) was lower than in municipalities with no heavy metal bands. Hospital admission rates were lower in municipalities with heavy metal bands compared with those with none. These associations could be explained partly by differences in the sociodemographic characteristics of residents in these municipalities. After adjustment for individual characteristics and area level cultural and economic characteristics-proportion of the population with no religious affiliation, unemployment rate, and per

\title{
WHAT IS ALREADY KNOWN ON THIS TOPIC
}

Heavy metal lyrics and lifestyle glorify risk taking, reckless behaviour, and intoxication; preference for heavy metal music has been reported to be correlated with antisocial behaviours, suicide, and drug misuse

Previous studies were biased by self-identification to particular music styles, cross sectional designs, and situational factors

\section{WHAT THIS STUDY ADDS}

Living in cities with a high density of heavy metal bands is not associated with higher hospital admission rates or mortality

Cities with a high density of heavy metal bands may have a small mortality advantage in terms of internal and alcohol attributable causes

The study results highlight the need to establish the mechanisms through which exposure to heavy metal and other music genres may be associated with health and mortality

capita expenditure on culture and education-large cities with a high density of heavy metal bands (8.2-11.2 per 10000) showed a mortality advantage (hazard ratio $0.92,95 \%$ confidence interval 0.88 to 0.96 ). In contrast, the association for hospital admission was fully attenuated (incidence rate ratio $0.99,95 \%$ confidence interval 0.92 to 1.06 ). The cause specific analysis showed similar results, with the association most pronounced for alcohol attributable mortality (hazard ratio $0.83,95 \%$ confidence interval 0.75 to 0.93 for cities with a high density of heavy metal bands) and alcohol attributable hospital admissions (incidence rate ratio 0.84, 95\% confidence interval 0.74 to 0.97 for cities with a high density of heavy metal bands) in the fully adjusted models. No association with heavy metal band density was found for the analysis using appendicitis as a negative control outcome.

\section{CONCLUSIONS}

The study found no evidence for adverse health outcomes with increasing density of heavy metal bands. Cities with a high density of heavy metal bands showed slightly lower rates of mortality and of hospital admissions for alcohol related problems and self-harm. Although residual confounding remains a problem in observational studies, vibrant local heavy metal scenes-comparable to many other forms of cultural capital-might help to promote health through healthier lifestyles, better coping mechanisms, and a stronger sense of community.

\section{Introduction}

Evidence suggests that physical, social, and demographic characteristics of regions are associated with health and cause specific mortality, and these associations may be independent of individual characteristics. $^{12}$ In particular, various measures of area level social cohesion, participation, and cultural involvement might be relevant to health. ${ }^{13}$ Although the emotional and spiritual effects of music are broadly acknowledged, evidence for the effects of different musical genres on health-both at the individual and area level-is largely missing or inconclusive.

Studies of music listeners and performers, at an individual level, provide conflicting results. Listeners of some music genres-such as classical music-are observed to experience reduced levels of stress. ${ }^{4}$ These findings are supported by experimental studies, which found positive effects of classical music (but also of other music genres) on heart rate variability and behaviour of kennelled dogs. ${ }^{5}$ Differences in heart rate and blood pressure before and after listening to classical or heavy metal music were not, however, 
observed in healthy human students. ${ }^{7}$ Preference for different genres of music is, however, associated with mental health status among adolescents, with an inclination for rap and heavy metal correlated with antisocial behaviours, suicidality, and drug use..$^{8-10}$ Furthermore, concert goers aged 16-29 are more prone to substance misuse than the general population of the same age. ${ }^{11}$ Similarly, robust evidence shows that mortality among popular music stars is higher than among matched controls, ${ }^{12}$ and that excess mortality is particularly high for violent deaths and liver disease. ${ }^{13}$

Although these studies have improved our understanding of the health effects of exposure to musical genres, such analyses may be vulnerable to selection biases because of self-identification to particular music and lifestyles. Much of the evidence on heavy metal is based on small samples, is cross sectional and self-reported, and the direction of effects, mechanisms, and moderators remains unclear. In addition, individual level studies cannot identify the broader effects of music cultures on the health of all community members. Although a previous study found an association between country music radio time and increased suicide rates across 49 US metropolitan areas, ${ }^{14}$ studies of area level heavy metal prevalence on health are lacking.

Lyrics and lifestyle associated with heavy metal often glorify risk taking, reckless behaviour, and intoxication. It is possible therefore that high environmental or community level exposure to heavy metal may increase health problems from accidental, violent, and alcohol attributable causes. Exposure to

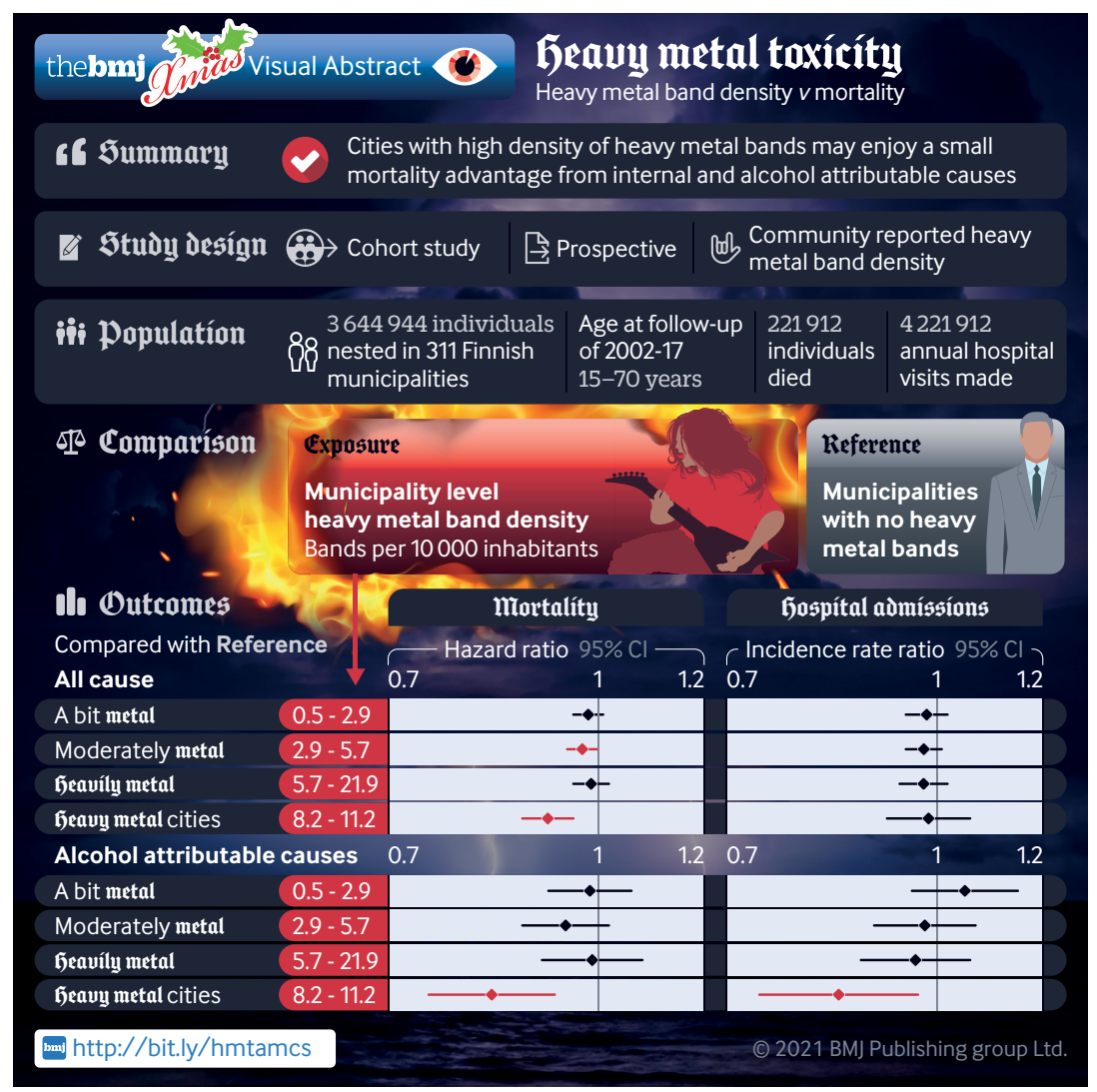

heavy metal might, however, have beneficial health effects by helping people cope with depression, buffering people against the fear of death, ${ }^{15}$ and providing solidarity and community. ${ }^{1617}$ Furthermore, many Finnish heavy metal songs draw inspiration from folklore and myths, ${ }^{18} 19$ invoking forests and nature; therefore, it can be hypothesised that heavy metal increases exposure to the health benefits of nature, green areas, and possibly even exercise.

We assessed the contribution of area level density of heavy metal bands on cause specific hospital admissions and mortality in Finland accounting for individual socioeconomic and family characteristics and area level cultural, social, and economic characteristics. Importantly, our measure of heavy metal prevalence does not only capture effects that are experienced by heavy metal fans but also effects that spill over to the whole community as fans interact with other community members in schools, work places, and families.

Finland is a unique setting for our study as it is known for being the heavy metal capital of the world..$^{20}$ In May 2016, President Obama observed in his White House welcome speech for the Nordic heads of state that "Finland has perhaps the most heavy metal bands in the world per capita, and also ranks high on good governance" and continued to deliberate whether this association was purely correlational. ${ }^{21}$ Finland also has large regional variations in heavy metal penetrance, allowing for robust area level analysis.

\section{Methods}

From individual level data compiled by Statistics Finland we obtained data on all people residing in Finland at the end of 2001. The data originate from various administrative registers and cover individual socioeconomic and personal characteristics, as well as hospital records with diagnoses and dates, and causes of death during 2002-17. Participants were followedup from the end of baseline year 2001 until the date of death or the last day of 2017. Emigrants were censored at the date of emigration. Children younger than 15 were excluded. We also censored participants at the end of the year in which they became 70. A total of 3644944 people contributed to the analyses.

\section{Measures}

Data on the number of heavy metal bands in each Finnish municipality were obtained from Encyclopaedia Metallum: The Metal Archives, which lists all metal bands in the world and has been used in other scientific studies on metal music. ${ }^{22}$ Registered users-commonly bands and fans-provide details of the band in the database. On entry of a band, a music sample is requested to verify the genre. Data reliability relies on editorial oversight of registered users and the database operator. From the database, which covered 2002-19, we identified 3871 metal bands based in Finland. Four hundred and nine (10.6\%) bands had not disclosed their municipality, and these were excluded. The data are freely available at www.metalarchives.com. 
As the main independent variable of interest, the number of metal bands per 10000 inhabitants was calculated using the total population of each municipality in 2018. Of 311 municipalities, 122 reported no metal bands. Therefore, we created a categorical variable for heavy metal band density so that the municipalities with no heavy metal bands formed one category and the rest were divided into tertiles for heavy metal band density. As bands tend to cluster in larger cities, ${ }^{22}$ the highest third was dominated by seven of the nine Finnish cities with a population greater than 100000 . We added a separate category of cities with a high density of heavy metal bands, and the remaining municipalities were divided into thirds, each consisting of 60 to 61 municipalities. Thus, our variable for heavy metal band density was classified as no heavy metal bands, three tertiles of heavy metal band density per 10000 inhabitants (0.52.9, 2.9-5.7, 5.7-21.9), and cities with high heavy metal band density per 10000 inhabitants (8.2-11.2).

Dates and causes of death were derived from the death register of Statistics Finland. For the cause specific analyses we identified four broad groups according to ICD-10 (international classification of diseases, 10th revision) codes: internal causes of death excluding alcohol attributable deaths (A00-R99), alcohol attributable diseases and unintended alcohol poisoning (F10, G31.2, G40.51, G62.1, G72.1, I42.6, K29.2, K70, K86.0, K85.2, O35.4, P04.3, Q86.0, X45), accidental and violent causes (V01-X44, X46-Y89), and suicides (X60-X84, Y87.0). Hospital admission dates and diagnoses originated from the hospital discharge records of the Finnish Institute for Health and Welfare. We calculated the number of years during follow-up in which the participant had at least one hospital visit for any cause and for specific causes. Additionally, we included a category for mental health diagnoses (F00-F99) excluding alcohol attributable diagnoses, and removed causes related to childbirth from the analysis (000-099).

We adjusted for individual level socioeconomic and personal characteristics obtained from the population registers of Statistics Finland. These variables were measured at the end of the baseline year 2001 and included sex (male, female); age; language (Finnish, Swedish, other); socioeconomic position (upper white collar, lower white collar, manual employee, self-employed, other); highest achieved educational qualification, categorised as basic (nine years of comprehensive school), intermediate (10-12 years of education), and high ( $\geq 13$ years); economic activity (employed, unemployed, retired, other); logarithm of individual disposable income; marital status (never married, married, divorced, widowed); living alone (alone, other); and housing tenure (owner, renter, other). To account for exposure to more physical forms of metal, we also included an individual level dummy variable indicating whether the individual was working in a metal processing occupation.

Municipal level data provided by Statistics Finland were based on aggregated individual level registers as well as information reported by municipal authorities. The municipal level data are freely accessible (www. stat.fi). Municipal level covariates that capture variation in economic and cultural characteristics of the municipalities and that are also associated with health included unemployment rate, proportion of residents not belonging to a religious community, proportion of residents living in urban areas, logarithm of per capita municipal expenditure on education and culture, and logarithm of net municipal public debt per capita.

To assess the association further, we analysed two negative control outcomes that are likely to be unrelated to exposure to heavy metal music: hospital admission resulting from the toxic effects of metals (ICD-10: T56) and appendicitis (ICD-10: K35). As fewer than 10 hospital admissions related to the toxic effects of metal were documented, we only report results for appendicitis $(n=61456)$. Additionally, we conducted subgroup analyses during follow-up for men and women and for the population aged 15-29 (younger people probably being more exposed to heavy metal music).

\section{Statistical analyses}

Cox proportional hazards models were used for mortality analysis, and for hospital outcomes we estimated incidence rate ratios using negative binomial models. To account for correlation within the municipalities, we clustered standard errors at the municipal level. Three models were used-model 1 was adjusted for age, sex, and language; model 2 was adjusted for all individual characteristics; and model 3 was adjusted for all individual and municipal level variables. All analyses were performed using Stata $16.1 .^{23}$

\section{Patient and public involvement}

Because this was a pseudonymised register based study of the total population of Finland and because of covid-19 restrictions, participants or the heavy metal community were not involved in setting the research question or the outcome measures, nor were they involved in developing plans for design or implementation of the study. Participants were not asked to advise on the interpretation or writing up of results.

\section{Results}

Areas without heavy metal bands were on average more religious, less urban, had lower unemployment, and invested more in culture and education (table 1). Age, sex, and language adjusted hospital admissions and death rates indicated lower all cause rates among those living in cities with heavy metal bands (table 2).

Age and sex adjusted regression models suggested that municipalities with a low or moderate density of heavy metal bands (two lowest tertiles) had 7-8\% lower mortality compared with municipalities with no heavy metal bands (fig 1, model 1). These differences, however, became attenuated after adjustment for individual 


\begin{tabular}{|c|c|c|c|c|c|}
\hline \multirow[b]{2}{*}{ Characteristics } & \multirow{2}{*}{$\begin{array}{l}\text { No heavy metal } \\
\text { bands }(n=122)\end{array}$} & \multicolumn{3}{|c|}{ Heavy metal band density per 10000 inhabitants } & \multirow{2}{*}{$\begin{array}{l}\text { Heavy metal band } \\
\text { cities }(n=7)\end{array}$} \\
\hline & & $0.5-2.9(n=61)$ & $2.9-5.7(n=61)$ & $5.7-21.9(n=60)$ & \\
\hline No (\%) of population total & $291595(8)$ & $473843(13)$ & $911236(25)$ & $911236(25)$ & $1057034(29)$ \\
\hline Proportion of population with no religious affiliation (\%) & 17 & 19 & 19 & 19 & 31 \\
\hline Mean culture and education expenditure per capita $(€)$ & 1957 & 1865 & 1860 & 1832 & 1875 \\
\hline Proportion of residents living in urban areas (\%) & 6 & 27 & 33 & 36 & 94 \\
\hline Unemployment rate (\%) & 11 & 10 & 11 & 12 & 13 \\
\hline Mean municipal public debt per capita $(€)$ & 2764 & 2696 & 2717 & 3026 & 3284 \\
\hline
\end{tabular}

level covariates (fig 1, model 2). After adjusting for all individual and municipal level covariates in the full model (fig 1, model 3), a mortality advantage for cities with heavy metal bands emerged, presenting with an $8 \%$ lower mortality compared with municipalities without heavy metal bands. This mortality advantage of heavy metal cities was driven by lower mortality in all specific cause-of-death groups in the fully adjusted models. In particular, the largest difference was observed in mortality from alcohol attributable causes, with about $17 \%$ lower mortality in heavy metal cities.

An even greater advantage of cities with heavy metal bands was observed for hospital outcomes. The age and sex adjusted incidence rate of presentations to hospital for all causes was 16\% lower in cities with heavy metal bands compared with municipalities with no heavy metal bands, a difference not attributable to individual characteristics (fig 2, models 1 and 2). In the model adjusting for all individual and municipality characteristics, however, the health advantage disappeared (fig 2, model 3). This attenuation was caused by adjusting for the proportion of non-religious population in particular (supplementary table 2, model 2a). In fully adjusted models, hospital admissions for alcohol attributable causes in cities with heavy metal bands (incidence rate ratio 0.84, 95\% confidence interval 0.74 to 0.97 ) and for intentional self-harm in the third tertile $(0.79,0.65$ to 0.96$)$ were lower than in the municipalities with no heavy metal bands.

Subgroup analysis for men and women showed similar associations in hospital admissions. Among the population aged 15-29, the results provided weak evidence for a higher rate of hospital admissions for mental disorders and self-harm, whereas other hospital outcomes showed an advantage for municipalities with heavy metal bands (supplementary table S3). The analysis using appendicitis as a negative control outcome showed no association with density of heavy metal bands (supplementary table S4).

\section{Discussion}

Municipalities with a high density of heavy metal bands showed slightly lower hospital admission rates and mortality when accounting for age, sex, and language of the individuals residing in these municipalities. These associations between heavy metal band density and health outcomes partly disappeared when accounting for other individual level characteristics-except for cities with a high number of metal bands per capita. The mortality advantage was not explained by cultural and economic characteristics of areas, including unemployment rate and the cultural and educational expenditure per capita in these cities. In contrast, area level characteristics-the higher proportion of non-religious population in particular-explained the lower rate of hospital admissions in heavy metal cities. The cause specific analysis showed roughly similar results, but the association between heavy metal band density and mortality was only pertinent for deaths by internal causes and alcohol attributable causes and for hospital admissions for causes specifically attributable to alcohol. The association was observed for both men and women as well as for younger residents for some causes of hospital admissions.

\begin{tabular}{|c|c|c|c|c|c|}
\hline \multirow[b]{2}{*}{ Variables } & \multirow[b]{2}{*}{ No heavy metal bands } & \multicolumn{3}{|c|}{ Heavy metal band density per 10000 inhabitants } & \multirow[b]{2}{*}{ Heavy metal band cities } \\
\hline & & $0.5-2.9$ & 2.9-5.7 & $5.7-21.9$ & \\
\hline \multicolumn{6}{|l|}{ Deaths } \\
\hline All causes $(n=221912)$ & 600 & 558 & 548 & 594 & 586 \\
\hline Accidents and violence $(n=31671)$ & 70 & 63 & 61 & 71 & 68 \\
\hline Internal causes $(n=163193)$ & 484 & 453 & 443 & 470 & 461 \\
\hline Alcohol attributable causes $(n=26445)$ & 60 & 58 & 58 & 66 & 68 \\
\hline Suicide and self-harm $(\mathrm{n}=11844)$ & 25 & 22 & 22 & 25 & 24 \\
\hline \multicolumn{6}{|l|}{ Hospital admissions } \\
\hline All causes $(n=4237807)$ & 10070 & 9370 & 8831 & 9450 & 8515 \\
\hline Accidents and violence $(n=603490)$ & 1337 & 1243 & 1195 & 1297 & 1157 \\
\hline Internal causes $(n=3400776)$ & 8396 & 7811 & 7327 & 7813 & 6992 \\
\hline Alcohol attributable causes $(n=196125)$ & 496 & 454 & 384 & 440 & 333 \\
\hline Suicide and self-harm $(n=38756)$ & 67 & 71 & 66 & 66 & 78 \\
\hline Mental health $(n=327304)$ & 625 & 572 & 554 & 615 & 628 \\
\hline
\end{tabular}




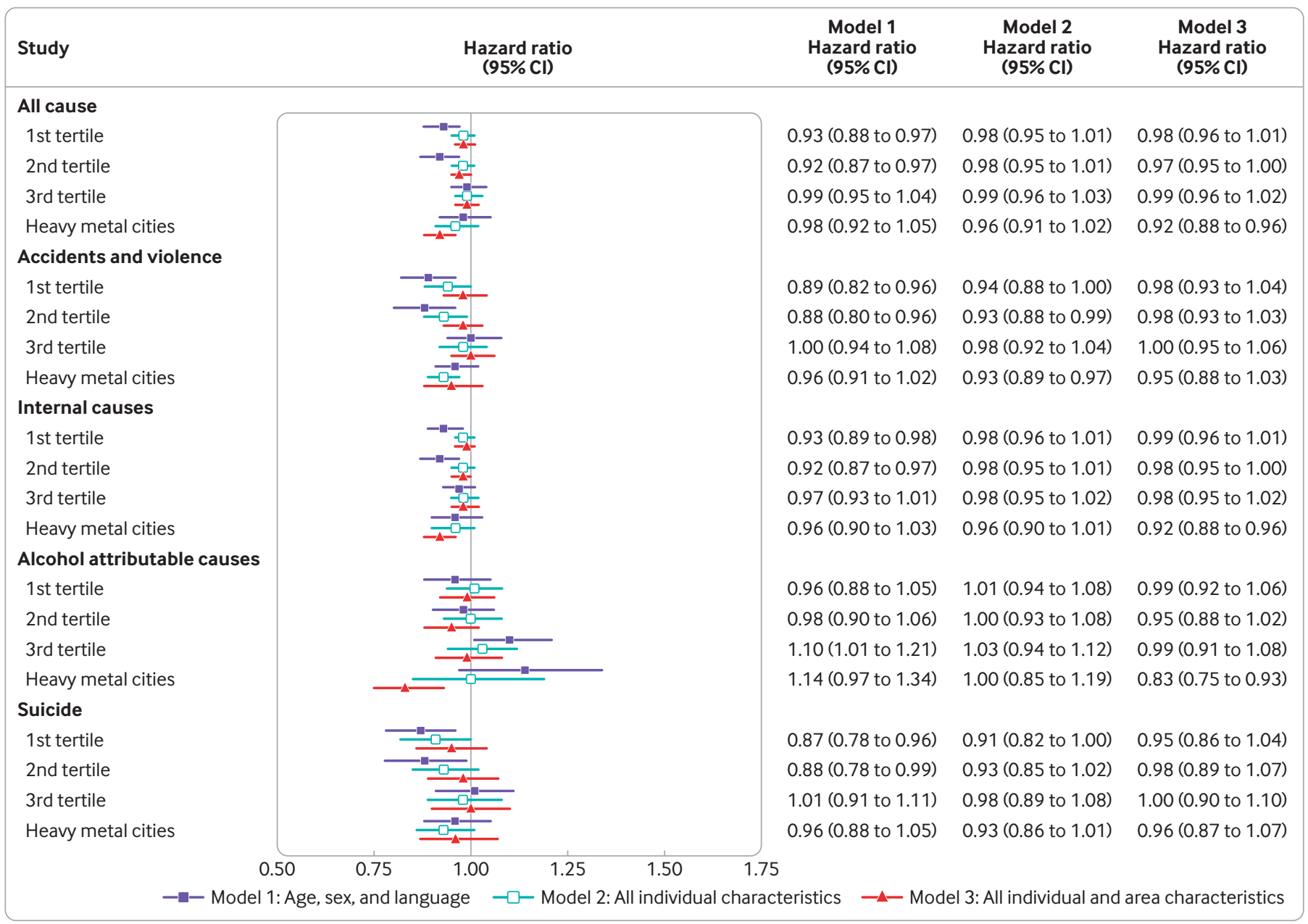

Fig 1 | Hazard ratios and $95 \%$ confidence intervals for all cause and cause specific mortality by heavy metal band density for Finnish residents aged 15-70 during 2002-17. The reference category is municipalities without heavy metal bands. Model 1 (Deep Purple square) adjusted for age, sex, and language. Model 2 (white square) further adjusted for all individual level characteristics, including socioeconomic position, education, economic activity, income, marital status, housing tenure, and metal worker status. Model 3 (red triangle) further adjusted for municipal level characteristics, including unemployment rate, proportion of population with no religious affiliation, proportion living in urban areas, municipal expenditure on education and culture, and municipal public debt

\section{Interpretation of results}

Our results indicate that the density of heavy metal bands might have a beneficial effect on adult health, especially in cities. It is difficult to determine the mechanisms of this association, but it could be partially explained by the beneficial effects of heavy metal music in coping with depression, and in improving social cohesion, a sense of community, and solidarity. ${ }^{15} 17$ The health advantage of cities with heavy metal bands appears to originate from alcohol attributable causes of death and hospital admissions. It is possible that factors related to the Finnish heavy metal scene and the connection of this music with myths and nature ${ }^{18} 19$ might have beneficial effects on health behaviours of residents in urban areas with heavy metal bands-for example, by encouraging exercise in forests, helping to avoid deleterious coping mechanisms such as substance misuse, and building and maintaining meaningful social bonds. The results also indicate that suicide, other violence, or mortality from accidental causes did not differ between areas with different heavy metal band densities. This is consistent with individual level evidence from France-based on an internet survey of 333 self-identified heavy metal fans-showing that the fans have similar or lower levels of anxiety and depression than the general population. The authors concluded that "contrary to critics who suggest that images of death and destruction in metal music have harmful consequences, the mental health of fans of this type of music is generally good." ${ }^{24}$ In fact, recent literature has argued that listening to extreme heavy metal music does not make angry people angrier but could represent a healthy and valuable social resource for those processing anger or in difficulty. ${ }^{16} 17$ It is tempting to speculate that similar processes could also be operating in the local community. The area level density of heavy metal bands might impact on health partly through its effects on heavy metal affiliates-the band members, fans, and venue organisers-as well as through the spillover effects on members of the broader community. We were unable to separate these effects because of the area level exposure variable 


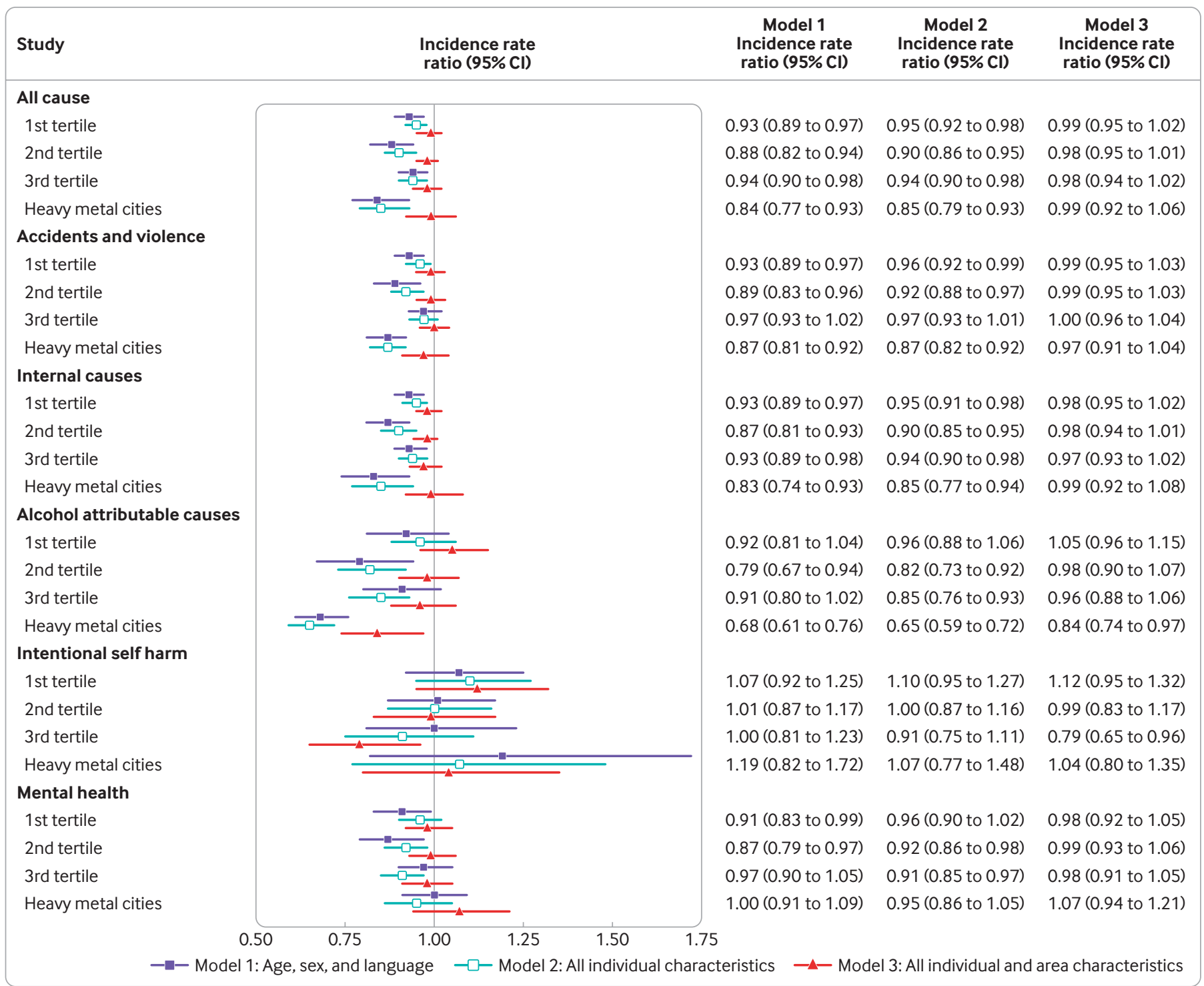

Fig 2 | Incidence rate ratios and 95\% confidence intervals for annual all cause and cause specific hospital admissions by heavy metal band density for Finnish residents aged 15-70 years during 2002-17. The reference category is municipalities without heavy metal bands. Model 1 (Deep Purple square) adjusted for age, sex, and language. Model 2 (white square) further adjusted for all individual level characteristics, including socioeconomic position, education, economic activity, income, marital status, housing tenure, and metal worker status. Model 3 (red triangle) further adjusted for municipal level characteristics, including unemployment rate, proportion of population with no religious affiliation, proportion living in urban areas, municipal expenditure on education and culture, and municipal public debt

used; therefore, we do not make strong claims about whether being a heavy metal fan is associated with the health outcomes.

It is also difficult to disentangle the health effects of one specific strain of culture-heavy metal musicfrom the general cultural environment. Different styles of music and other cultural pursuits do not exist in isolation. Heavy metal bands are likely to spring up in an environment that also fosters other cultural activities, and, vice versa, community involvement in heavy metal music might also benefit other music and cultural activities. Thus, the better health of inhabitants of cities with heavy metal bands might also be the results of other, causally more relevant area level characteristics being correlated with density of heavy metal bands. The hospital admission and mortality advantage of cities with heavy metal bands did not, however, emerge because of greater public investments in culture and education or low public debt. To the extent that low public debt reflects good governance, it seems that President Obama's tentative hypothesis on the association between good governance and density of heavy metal music ${ }^{21}$ does not hold. In the general media, the high prevalence of heavy metal bands in the Nordic countries has been described as the product of a countercultural reaction to affluence ${ }^{25}$; and the possibility thus emerges that the health advantage of cities with heavy metal bands simply reflects this affluence. This intriguing hypothesis does not receive support from our data, however, as the municipality unemployment rate was higher in cities with a high density of heavy metal bands. 
Finally, our analyses showed that an advantage in alcohol related mortality in cities with heavy metal bands emerged after adjustment for the proportion of non-religious population. For hospital admissions, the effect of adjustment for community level religiosity was the opposite. The exact mechanisms for these seemingly contradictory findings cannot be easily established. However, extending previous evidence, ${ }^{26}$ it could be that in more religious communities a higher proportion of people experience alcohol attributable health problems but lower mortality from these health problems than those living in communities with a lower level of religious affiliation (see also supplementary tables S5 and S6). It could even be hypothesised that in communities where religiosity and heavy metal coexist, support and access to help is better for those who misuse alcohol. Overall, although the exact mechanisms remain unknown, it seems possible that living in areas with vibrant metal music (or other music) communities is not health damaging but might actually be health promoting.

\section{Strengths and limitations of this study}

Our dataset was based on the total population of Finland, without non-participation, losses to followup, or self-reporting bias associated with heavy metal affiliation or reporting of health outcomes. The register based data enabled consistent measurement of sociodemographic characteristics, hospital admissions, and mortality, and linkage to a unique area level database of information on heavy metal. In interpreting our findings, however, three caveats common to all studies that assess the effects of area characteristics on health outcomes need to be considered. Firstly, although the data setup allows for a reliable estimation of the magnitude of area effects, with adjustment for several individual sociodemographic factors, our estimation might be compromised by inadequate measurement at both levels. Residual confounding and change in individual characteristics during the relatively long follow-up period cannot be excluded as an explanation for the observed area effects. In particular, the inability to account for selective migration could lead to biased area effects, because selective migration affects the composition of the area population and the area level variables themselves. The extent and direction of this bias is not well understood, but in the Finnish context this has been estimated to be moderate at best, ${ }^{27}$ and we have no evidence to believe that migration choices are strongly motivated by music preferences. In addition, some potentially relevant characteristics of the communities that are correlated with heavy metal band density could have remained unmeasured. If the association emerged because of an uncontrolled confounder, however, the same associations would likely have emerged also for appendicitis.

Secondly, our analyses could be based on area units that do not capture the right level of regional variability. Further assessment of different area units will likely lead to a better understanding of the mechanisms involved. Previous analyses of Finnish data indicate that the choice of area level does not create important bias. ${ }^{28}$ Our area level choice is justified by the fact that municipalities are responsible for the organisation of healthcare services as well as primary education and social and cultural programmes. Future studies should aim to establish whether the density of heavy metal bands in smaller neighbourhoods within urban areas is associated with health.

Finally, treating exposure as time varying could improve interpretation, although currently it is difficult to obtain reliable longitudinal data from the Metal Archives. Exposure to heavy metal music is unlikely to have remained stable over time, indicated by the decline in Google searches for "heavy metal" over the past 17 years in Finland (and globally). It is unfortunate that regional data on Google searches are incomplete in Finland, as we could have used these for confirmatory analysis (https://trends.google. $\mathrm{com} /$ trends/explore?date $=$ all\&geo $=$ FI\&q $=$ heavy $\% 20$ metal). Attempts to assess changes in exposure to heavy metal music in future studies are welcome. An interesting case study would be to evaluate the health consequences of the 2006 Eurovision song contest win by the band Lordi in its home town Rovaniemi, using abrupt shifts in time series or instrumental variables techniques.

Since the emergence of Black Sabbath in the early 1970s, heavy metal has developed many subgenres. Further analyses could aim to establish whether subgenres (although difficult to define exactly) are a fruitful target for study. Examples of these might include death metal or grindcore, which are often associated with deep vocals, extreme speed, ultimate expressions of heaviness, and lyrics associated with death, stylised violence, or mythology, as well as broader social and political issues (song titles eg, Death Certificate, ${ }^{29}$ Slowly We Rot, ${ }^{30}$ or Make Them Suffer ${ }^{31}{ }^{32}$; see lyrics for Death Certificate at https://www.azlyrics.com/ lyrics/carcass/deathcertificate.html). Overall, more research is needed to establish how any music genre is associated with the health of fans and the broader community. Such analyses could aim to replicate the results of our study in other countries or contextsfor example, in Sweden, another heavyweight on the heavy metal scene-and help to evaluate the specificity and generalisability of our findings.

\section{Conclusions}

Commenting on the state of the heavy metal landscape, the metal band Manowar has declared that "Other bands play... Manowar kill." The current evidence seems to confirm this, as it suggests that living in areas characterised by a high density of heavy metal bands (other than Manowar) is not associated with higher mortality. On the contrary, residents of these areas seem to enjoy a small health advantage. The results highlight the need to rigorously study the possible biological, behavioural, and social mediators to establish the mechanisms through which heavy metal or other music genres might be associated with health. 
We thank Olle Lundberg for drawing our attention to the website https://www.capitalofmetal.fi/home, and Elina Hiltunen for help with the graph.

Contributors: PM conceived the study with KK and LT. All authors participated in designing the study, generating hypotheses, and interpreting the data. All authors critically revised the manuscript for important intellectual content. PM and KK conducted the literature review and wrote the first draft of the manuscript. LT analysed the data. The corresponding author attests that all listed authors meet authorship criteria and that no others meeting the criteria have been omitted. PM is the guarantor.

Funding: PM was supported by the Academy of Finland (\#308247, \# 345219). PM has also received funding from the European Research Council under the European Union's Horizon 2020 research and innovation programme (grant agreement No 101019329). The study does not necessarily reflect the Commission's views and in no way anticipates the Commission's future policy in this area. The funders had no role in the study design, data collection and analysis, decision to publish, or preparation of the manuscript.

Competing interests: All authors have completed the ICMJE uniform disclosure form at www.icmje.org/disclosure-of-interest/ and declare: support from the Academy of Finland and European Research Council for the submitted work; no financial relationships with any organisations that might have an interest in the submitted work in the previous three years, no other relationships or activities that could appear to have influenced the submitted work.

Ethical approval: This study was approved by Statistics Finland board of ethics (TK-53-1490-18).

Data sharing: Owing to data protection regulations of the national register holders providing the data, we are not permitted to make the data available to third parties. Interested researchers should contact the register holding public institutions: Statistics Finland (www.stat.fi/tup/mikroaineistot/index_en.html), contact by email tutkijapalvelut(at)stat.fi; and Findata (https://findata.fi/en/), contact by email info(at)findata.fi.

The lead author (PM) affirms that the manuscript is an honest, accurate, and transparent account of the study being reported; that no important aspects of the study have been omitted; and that any discrepancies from the study as planned (and, if relevant, registered) have been explained.

Dissemination to participants and related patient and public communities: We aim to disseminate the results of the research to the general public and the heavy metal community through social media platforms and the Metal Archives. We will also communicate these results to Finnish policy makers, who decide upon the support for local heavy metal and other music communities, as well as to Population Europe (https://population-europe.eu) and the office of Barack and Michelle Obama.

Provenance and peer review: Not commissioned; externally peer reviewed.

This is an Open Access article distributed in accordance with the Creative Commons Attribution Non Commercial (CC BY-NC 4.0) license, which permits others to distribute, remix, adapt, build upon this work non-commercially, and license their derivative works on different terms, provided the original work is properly cited and the use is noncommercial. See: http://creativecommons.org/licenses/by-nc/4.0/.

1 Blomgren J, Martikainen P, Mäkelä P, Valkonen T. The effects of regional characteristics on alcohol-related mortality-a register-based multilevel analysis of 1.1 million men. Soc Sci Med 2004:58:2523 35. doi: 10.1016/i.socscimed 2003.09.027

2 Meijer M, Röhl J, Bloomfield K, Grittner U. Do neighborhoods affect individual mortality? A systematic review and meta-analysis of multilevel studies. Soc Sci Med 2012;74:1204-12. doi: 10.1016/j. socscimed.2011.11.034

3 Kawachi I, Kennedy BP, Lochner K, Prothrow-Stith D. Social capital, income inequality, and mortality. Am J Public Health 1997;87:14918. doi: 10.2105/AJPH.87.9.1491

4 Fancourt D, Williamon A. Attending a concert reduces glucocorticoids, progesterone and the cortisol/DHEA ratio. Public Health 2016:132:101-4 doi: 10.1016/i.puhe.2015.12.005

5 Bowman A, Dowell FJ, Evans NP, Evans NP, Scottish Spca. 'Four Seasons' in an animal rescue centre; classical music reduces environmental stress in kennelled dogs. Physiol Behav 2015;143:7082. doi: 10.1016/j.physbeh.2015.02.035
6 Bowman A, Dowell FJ, Evans NP, Evans NP, Scottish SPCA. 'The effect of different genres of music on the stress levels of kennelled dogs'. Physiol Behav 2017;171:207-15. doi: 10.1016/j. physbeh.2017.01.024

7 Kalinowska A, Kulakowska A, Kulak W, Okurowska-Zawada B. Effects of classical and heavy metal music on the cardiovascular system and brain activity in healthy students. Preliminary report Wpływ muzyki klasycznej i heavy metal na czynność układu krążenia i mózgu u zdrowych studentów. Doniesienie wstęne. Neurol Dziecięca 2013;22:17-22.

8 Baker F, Bor W. Can music preference indicate mental health status in young people?Australas Psychiatry 2008;16:284-8. doi: $10.1080 / 10398560701879589$

9 Scheel KR, Westefeld IS. Heavy metal music and adolescent suicidality: an empirical investigation. Adolescence 1999;34:253-73.

10 Hogan M, Bar-on M. Council on Communications and media. Impact of music, music lyrics, and music videos on children and youth. Pediatrics 2009;124:1488-94. doi: 10.1542/peds.2009-2145

11 Lim MS, Hellard ME, Hocking JS, Aitken CK. A cross-sectional survey of young people attending a music festival: associations between drug use and musical preference. Drug Alcohol Rev 2008;27:439-41. doi: 10.1080/09595230802089719

12 Bellis MA, Hennell T, Lushey C, Hughes K, Tocque K, Ashton JR. Elvis to Eminem: quantifying the price of fame through early mortality of European and North American rock and pop stars. J Epidemiol Community Health 2007;61:896-901. doi: 10.1136/ jech.2007.059915

13 Kenny DT, Asher A. Life expectancy and cause of death in popular musicians: is the popular musician lifestyle the road to ruin? Med Probl Perform Art 2016:31:37-44 doi: 10.21091/mppa.2016.1007

14 Stack S, Gundlach J. The effect of country music on suicide. Soc Forces 1992;71:211-8. doi: 10.2307/2579974.

15 Kneer J, Rieger D. The memory remains: How heavy metal fans buffer against the fear of death. Psychol Pop Media Cult 2016:5:258-72. doi: $10.1037 / \mathrm{ppm} 0000072$

16 Sharman L, Dingle GA. Extreme metal music and anger processing. Front Hum Neurosci 2015;9:272. doi: 10.3389/fnhum.2015.00272

17 Baker C, Brown B. Suicide, Self-harm and survival strategies in contemporary heavy metal music: a cultural and literary analysis. J Med Humanit 2016;37:1-17. doi: 10.1007/s10912-014-9274-8

18 Kärki K. Forging metal: The Kalevala in the Finnish heavy metal performance. 2015. https://www.academia.edu/20004163/ Forging_Metal_The_Kalevala_in_the_Finnish_Heavy_Metal_ Performance

19 Tolvanen H. The quiet past and the loud present: the Kalevala and heavy metal. Vol Rev Musiques Pop, 2006, 10.4000/volume.498.

20 A world map of heavy metal density. Big Think. 2012. https://bigthink. com/strange-maps/560-a-world-map-of-heavy-metal-density

21 Obama B. Remarks by President Obama, President Niinistö of Finland, and Prime Minister Solberg of Norway at the Nordic Leaders' Summit arrival ceremony. The White House 2016. https:// obamawhitehouse.archives.gov/the-press-office/2016/05/13/ remarks-president-obama-president-niinist\%C3\%B6-finland-andprime-minister

22 Makkonen T. Tales from the thousand lakes: placing the creative network of metal music in Finland. Environ Plann A 2014;46:1586600. doi: 10.1068/a130020p.

23 StataCorp. Stata Statistical Software: Release 15. StataCorp LLC, 2017.

24 Recours R, Aussaguel F, Trujillo N. Metal music and mental health in France. Cult Med Psychiatry 2009;33:473-88. doi: 10.1007/ s11013-009-9138-2

25 Florida R. How heavy metal tracks the wealth of nations. Bloomberg 2014. https://www.bloomberg.com/news/articles/2014-05-26/ how-heavy-metal-tracks-the-wealth-of-nations

26 Idler E, Blevins J, Kiser M, Hogue C. Religion, a social determinant of mortality? A 10-year follow-up of the Health and Retirement Study. PLoS One 2017;12:e0189134. doi: 10.1371/journal. pone.0189134

27 Martikainen P, Sipilä P, Blomgren J, van Lenthe FJ. The effects of migration on the relationship between area socioeconomic structure and mortality. Health Place 2008;14:361-6. doi: 10.1016/j. healthplace.2007.07.004

28 Tarkiainen L, Martikainen P, Laaksonen M, Leyland AH. Comparing the effects of neighbourhood characteristics on all-cause mortality using two hierarchical areal units in the capital region of Helsinki. Health Place 2010;16:409-12. doi: 10.1016/j.healthplace.2009.10.008

29 Carcass. Death Certificate. United Kingdom: Earache 1993.

30 Obituary. Slowly We Rot. US: R/C Records 1989

31 Cannibal Corpse. Make Them Suffer. Metal Blade, 2006.

32 O'Neill A. A history of heavy metal. Headline, 2017.

Supplementary materials: Additional tables S1-3 